\title{
DESAFÍOS DEL DESARROLLO EN LA provincia de Río Negro, Argentina
}

\author{
Paula Gabriela Núñez, ${ }^{a}$ Carolina Lara Michel ${ }^{\mathrm{b}}$ y Santiago Conti ${ }^{\mathrm{b}}$
}

Fecha de recepción: 19 de febrero de 2020. Fecha de aceptación: 15 de junio de 2020.

$$
\text { http://doi.org/10.22201/iiec.20078951e.2020.203.69581 }
$$

Resumen. El artículo explora el desarrollo rural en la región norpatagónica andina de la provincia de Río Negro, Argentina. Analiza un área ambiental adecuada para un desarrollo rural extensivo que no termina de integrarse como área productiva. Vincula las dificultades del presente a contradicciones estructurales de su incorporación regional a las administraciones nacional y provincial. Indaga los términos significativos que marcaron las políticas territoriales observando el modo en que las mismas consideraron a los habitantes de la región y sus actividades. Expone cómo los límites a las dinámicas de integración se sostienen en modelos de crecimiento que, desde las nociones de progreso, desarrollo e innovación, han omitido reconocimientos a los actores productivos locales. Palabras clave: desarrollo rural; progreso; innovación técnica; región norpatagónica andina; integración territorial; política económica.

Clasificación JEL: N96; O18; R11; R58.

\section{Development Challenges in the Province of Río Negro, Argentina}

\begin{abstract}
This article examines rural development in the North Andean region of Río Negro province, Argentina. The authors analyze an environmental area suitable for extensive rural development that is not fully integrated as a productive area. Additionally, this article associates present difficulties with structural contradictions inherent in its regional incorporation to the national and provincial administrations. It then investigates the significant terms that characterized territorial policies, while illuminating how these terms viewed the inhabitants of the region and their activities. Finally, the article goes on to expose how the limits to the dynamics of integration are sustained by growth models that, based on notions of progress, development, and innovation, have overlooked local productive actors.
\end{abstract}

Key Words: rural development; progress; technical innovation; northern Andean region; territorial integration; economic policy.

\footnotetext{
${ }^{a}$ Universidad de Los Lagos, Osorno, Chile; Universidad Nacional de Río Negro; Instituto de Investigación en Diversidad Cultural y Procesos de Cambio (IIDyPCA); Conicet, Argentina. b Universidad Nacional de Río Negro, Argentina. Correos electrónicos: paula.nunez@ulagos.cl; cmichel@unrn.edu.ary santiago.conti@gmail.com, respectivamente. Se agradece el trabajo de revisores anónimos. Esta publicación se realizó con el apoyo de la Universidad de Los Lagos y en el marco de resultados tomados por el PIP 0838 .
} 


\section{INTRODUCCIÓN}

El presente artículo cuestiona el desarrollo rural en la región andina argentina de la provincia de Río Negro y se ubica en los estudios sobre el desarrollo rural que se han propuesto, tanto desde términos económicos y multicausales (Garcés, 2019), como desde otros que reparan la forma en que tales perspectivas se transforman en políticas de intervención (Lattuada et al., 2015). En este caso en particular, se busca incorporar un carácter geográfico e histórico en la pregunta por el desarrollo (Delgadillo, 2009), evidenciando la necesidad de considerar la forma en que se integran las regiones, ya que se toma como caso un área marginal (Serje, 2005), con marcas que evidencian esta condición en las políticas de desarrollo local (Herrera y Herrera, 2014; Núńez, 2016).

La literatura existente reconoce una integración desigual del territorio patagónico que contiene a la provincia de Río Negro. Expone permanencias de dinámicas de colonialismo interno tanto en la Patagonia argentina como en la chilena (Navarro, 2011; Núñez et al., 2017; Klubock, 2014). El caso de estudio agrega a ello el hecho de que se trata de una región aledańa a la frontera con Chile (Bessera, 2008).

De tal forma que se explorarán dinámicas productivas en una región afectada por las desigualdades territoriales, que se evidencian en restricciones al acceso a derechos, a servicios básicos; así como también a limitantes en términos productivos, de infraestructura y de comunicación (Herrera y Herrera, 2014).

Se buscó abordar ciertos aspectos caracterizados como particularidades que responden a dinamismos de integración territorial más amplios, tanto en espacio como en tiempo, y que impactan en el reconocimiento de la producción local y los actores dedicados a la misma.

Dentro del mapa rionegrino, se analizan condicionantes y características de una región escasamente integrada al orden provincial, la zona andina (véase figura 1) a través de las políticas económicas delineadas para sus dos localidades más emblemáticas: San Carlos de Bariloche, localizada a orillas del lago Nahuel Huapi, y El Bolsón ubicada a 130 kilómetros al sur.

Para exponer los desafíos del desarrollo presente, en una primera parte se recorrerán los escritos que problematizan la producción regional actual y la integración económica del espacio en la actualidad. Sumado a ello un análisis de formularios censales agropecuarios diseńados por el Estado nacional. En una segunda parte, se interpelará el proceso actual a partir de debates contemporáneos surgidos de actores locales reunidos para reflexionar sobre este punto. ${ }^{1}$ En

1 En los cursos "Epistemología y Metodología de la Investigación Científica”, INTA Bariloche, en 2018, con extensionistas de norpatagonia y "Modelos de desarrollo y ruralidad en norpatagonia", 
una tercera parte, se presenta el desarrollo local analizado históricamente, en busca de explorar los sentidos de tres términos emblemáticos de documentos gubernamentales: "progreso", "desarrollo" e "innovación". Cerrando el escrito con una reflexión sobre la relevancia de considerar estas variables en los estudios económicos posteriores.

\section{LOS ANDES RIONEGRINOS}

La provincia de Río Negro, en Argentina, corresponde al área norpatagónica, región que posee una alta diversidad biogeográfica. Los Andes, en particular, son una región de montaña con grandes paisajes de bosques y lagos, que históricamente se destinaron a actividades ganaderas y agrícolas (Méndez y Muñoz, 2013).

Una de las características de este espacio es la incidencia del turismo como actividad emblemática que, en Bariloche, a partir de la década de los treinta desplazó la actividad agrocomercial de principios del siglo $\mathrm{xx}$, con procesos similares a los que en la actualidad se registran en la localidad de El Bolsón (Cobelo, 2017). Así, Bariloche remite a la temprana incorporación de un área natural protegida que alojó dinámicas diferentes del resto del territorio rionegrino y del país (Oglietti y Colino, 2011), y tensiona la posibilidad misma de concebir la producción rural como parte significativa de las iniciativas locales. Mientras que la especificidad de El Bolsón focaliza la pregunta por la producción rural, ligada sobre todo al lúpulo y la fruta fina.

La norpatagonia se integra tardíamente al concierto nacional, pero cuando lo hace, se menciona el destino natural de toda la región para actividades agrícolas, aún sin haber relevado el espacio (Núñez y Lema, 2018). A pesar de reconocerse un importante comercio transcordillerano de la producción andina y de la estepa norpatagónica (Méndez y Muñoz, 2013), la región no se toma como área significativa del desarrollo agrícola nacional.

Una de las producciones más significativas de la zona, la de fruta fina, da elementos para reconocer la carga paradojal de la actual producción en la región. La producción de fruta fina se instala como identidad en la región en la que incluso se festeja la "Fiesta de la Fruta Fina". Los registros regionales ubi-

UNRN-El Bolsón, en 2018, con investigadores de temáticas rurales de la zona, se suma el encuentro de creación del "Observatorio de Políticas Públicas" en El Bolsón en 2018, que reunió más de 50 productores(as) rurales, investigadores, autoridades políticas y docentes en torno al desafío del ordenamiento local. 





can los inicios de la actividad productiva en la década de los sesenta, cuando se plantea su comercialización (Méndes, 2010). Lo anterior contrasta con el reconocimiento, dentro de las mismas fuentes, que indican antecedentes en la década de los cuarenta sobre la generalización de su uso doméstico. Se puede decir que, en los estudios académicos actuales, la visibilidad de la actividad depende de su posibilidad de comercialización en mercados formales.

La observación sobre la actividad económica reconoce la salida al mercado como ítem excluyente para dar cuenta de una actividad que se reconoce anclada en dinamismos familiares e informales, al punto que, los estudios sobre el desarrollo y la organización territorial regional en la zona andina elaboran temporalidades ligadas a salidas a mercados, omitiendo elementos y redes de producción familiar (Méndes, 2010; Danklmaier et al., 2013; Valtriani, 2008).

Como ejemplo de esta tensión cabe analizar el informe de Danklmaier et al. (2013), que avanza en un análisis foda (Fortalezas, Oportunidades, Debilidades, Amenazas) a partir de reuniones con los principales actores productivos de la región. Los autores observan la producción de fruta fina que se describe como una de las más emblemáticas de la zona. Mencionan que la calidad de la producción local es superlativa respecto del resto del país, que su comercialización se da desde una valoración al producto que tiene que ver con la identidad local, pero a pesar de todo esto, señalan que "la producción 'casera' de procesados mueve un volumen importante de producto en el mercado informal. No existen actualmente registros estadísticos de este sector" (Danklmaier et al., 2013, p. 34), haciendo referencia a que tampoco existe un conocimiento de la producción denominada de "autoconsumo", a pesar de la amplia red de intercambio entre productos domésticos, como parte de la traza de informalidad, reconocida como parte de la trama de resiliencia en un marco de inestabilidad que se presenta como característica de la zona (Cobelo, 2017; Danklmaier et al., 2013).

Los estudios explicitan la invisibilidad que resulta impuesta por tomar la comercialización formal como medida de la existencia. Este aspecto se reitera en la producción hortícola, que se describe como especialmente relevante y usual a escala doméstica (Danklmaier et al., 2013), pero casi ausente en términos comerciales, o en la silvicultura, donde se indica que "[...] existe una silvicultura con un enfoque social, a partir de la participación de la comunidad, con una visión sistémica del bosque y las plantaciones, en cuanto a la toma de decisiones sobre la gestión de sus recursos naturales, con la integración de la familia como núcleo de la empresa y con un fuerte componente de género. Estas experiencias son muy locales y no se han articulado a nivel territorial" 
(Valtriani, 2008, p. 278), y también en la silvestría, como la recolección de hongos o de frutos de especies nativas, como el sauco, de enorme importancia en la economía doméstica y asociada a algunos de los productos más valorados de la región.

La paradójica sobredimensión del mercado como fuente de respuestas frente a las contradicciones de la economía local se repite en la pregunta por producciones con más de 70 años en la región. Las mismas existen sólo si salen al mercado, más allá de su historia o relevancia social.

Pero estas paradojas de las miradas locales se suman a otras originadas en una escala más amplia. Una de las características productivas de esta región es que su producción tiene un reconocimiento menor que el de otras regiones. Esto puede observarse a través del modo en que se construyen los indicadores del Censo Nacional Agropecuario (CNA). Cabe señalar que el censo es una de las principales tecnologías de ordenamiento del Estado (Otero, 1999). En este caso, los censos nacionales agropecuarios refieren a la principal base productiva del país. Una característica que se reitera en estos censos, como base del conocimiento productivo, es la omisión sistemática de aquello que no sale al mercado (Michel et al., 2020), lo que lleva a un problema general. Las unidades productivas de agricultura familiar, en la Patagonia, son muy numerosas. En la zona que se estudia, casi el total de la población se localiza en una dimensión de pequeña escala, lo que muestra que la dinámica familiar y de autoconsumo es especialmente dinámica. Conti y Núñez (2012) reconocen que el autoconsumo en la zona de El Bolsón no sólo refiere a lo que se come en la unidad productiva, sino a un amplio ejercicio de intercambios informales que, esporádicamente, se transforman en venta comercial, estableciendo modos no monetarios relacionados al destino de la producción que no por ello dejan de ser relevantes. Sin embargo, el cNA no toma datos al respecto y los productores reunidos, cuando debaten el tema, tampoco lo consideran en tanto no existan indicadores oficiales que permitan esta caracterización.

Como consecuencia, el financiamiento se dirige a los sectores más relevados (productores más capitalizados con producciones orientadas a la exportación), que se ubican fuera de la región de estudio. En la provincia de Río Negro las especies sobre las que se preguntan más a detalle son las frutas de pepa, como la manzana y la pera, que se cultivan en Alto Valle y Valle Medio (véase figura1). Mientras que para aquellos donde se toman menos datos, como son las que se cultivan en la región andina, la medida de la ignorancia parecería ser el fundamento de la falta de financiamiento. Así, la omisión del reconocimiento no es sólo una paradoja que desacredita los pilares identitarios locales, es una traba al acceso de financiamiento y a la posibilidad de gestión. 
La fruta fina, por ejemplo, se releva en dos variables. Los censos contemplan 84 variables para caracterizar la producción de manzana y 52 variables para caracterizar la producción de pera. La cantidad de información relevada para la manzana y la pera tiene, como contracara, el desinterés por conocer factores que podrían ser significativos en producciones que no son de exportación. Otro ejemplo es el de las hortalizas, que sólo se relevan por superficie de producción y no por kilos producidos, lo cual la muestra como complementaria.

De los censos parecería desprenderse que el potencial del cambio se vincula a una estructura donde el mercado exterior parecería ser la referencia ineludible de la producción rural, con centros comerciales que además se establecen por fuera de la zona andina.

Esto lleva a desafíos que tienen que ver con las variables con que se mide el desarrollo. En tanto la actividad siga siendo la de exportación, los cambios de innovación tecnológica van a seguir buscando vías de acceso a un mercado que desconoce el enorme potencial productivo basado en tramas locales, a pesar de que la promesa del desarrollo es la mejor calidad de vida de la población en general.

Existe un foco que todavía no termina de tomar la economía regional. El intercambio solidario ha sido la respuesta central frente a fenómenos como explosiones volcánicas, incendios domésticos, nevadas, inundaciones, incluso frente a la hiperinflación (Lacoste, 2003), en toda la región. El trueque se profundizó durante las crisis (Colino et al., 2016). Un ejemplo es 2002, cuando el CNA se enfrenta a las consecuencias regionales de la crisis económica nacional de 2001. Ese año no se tomaron datos de la región porque nadie vendía con moneda oficial. Entonces, ¿no hubo producción? De hecho sí, pues se dieron amplios intercambios, apoyados muchas veces en nueces devenidas en moneda social de las zonas rurales, que resultaron dinamizadores de la producción local y que dieron las condiciones para la permanencia. ${ }^{2}$ Queda, entonces, abierta la cuestión de si la opacidad reconocida en el censo se repite o se modifica en las formas de medición de las actividades en la región, así como en la intervención técnica que se planifican para la actividad. 


\section{LA INTERVENCIÓN TÉCNICA EN PRÁCTICAS INVISIBLES}

El modo de medir y reconocer deviene en prácticas de intervención técnica. Río Negro, como provincia, se adhirió tempranamente a los programas de desarrollo nacional, tomando la innovación como horizonte (Lattuada et al., 2015), pero existen dinámicas de pervivencia de desigualdad que parecen trascender las propuestas de cambio. Los programas repiten, como los censos, la sobreestimación de lo comercial, y por ello, las herramientas de promoción tecnológica están mediadas por ese objetivo.

Los agentes de extensión reconocen la existencia de un conocimiento local con características holísticas, con dinámicas vinculares y afectivas, centrales pero invisibles. ${ }^{3}$ El objetivo del mercado se descubre como disruptor en lugar de articulador o promotor, pues la mejora en la comercialización no implica mejor integración laboral, o siquiera integración a secas.

Los técnicos "descubren" la sobreestimación del mercado en la idea de "mejora" y de "competitividad" que se plantea en los planes de desarrollo (prosap, 2011), desde los cuales deben diseñar su intervención orientada a una mejor inserción al mercado. Pero algo más, descubren que esa sobreestimación se apoya en un modelo ideal y ajeno de producción, uno de los participantes del curso sobre "Epistemología y Metodología de la Investigación Científica" señaló que sin conocer si lo que se hace en este sistema realmente necesita una mejora o es lo mejor que existe para ese contexto. ${ }^{4}$ El modelo no demanda el estudio de lo local, sino que se plantea como el deber-ser para lo local. Ejemplo de ello son las mejoras genéticas propuestas sin tener en cuenta el ambiente productivo, o los manejos del sistema que en ocasiones resultan poco prácticos e ineficientes por el uso de insumos, que el productor no sabe utilizar o le cuesta conseguir, como el uso de alimentos no convencionales o mecanismos para ampliar la base forrajera.

Los especialistas consultados dentro de los encuentros tomados como referencia, plantearon que los saberes locales son apartados, generando una cadena de producción aceitada para responder a un mercado temporal o permanente que muchas veces genera daños en quienes lo toman y desplazamiento en quienes lo dejan. ${ }^{5}$ En la cadena de producción se puede ver que, desde el sector primario hacia adelante, participan un conjunto de profesionales, tanto

\footnotetext{
Cita tomada del curso "Epistemología y Metodología de la Investigación Científica".

Cita tomada en curso de posgrado "Epistemología y Metodología de la Investigación Científica". Idem.
} 
del sector privado como del sector público, que buscan atender no sólo las necesidades, pues buscan asegurar la calidad agroalimentaria de la población. Se apelan a intervenciones diseñadas desde la genética de animales, la sanidad, el manejo predial y la promoción de actividades intensivas, donde la innovación es la matriz de abordaje.

En un encuentro específico para reflexionar sobre la producción ovina, R., ${ }^{6}$ uno de los participantes, mencionaba que, quienes estaban en el campo veían en productores y cabañeros un conocimiento profundo, pero este "ver" dependía de la sensibilidad del técnico, en un contexto que R. consideraba tensionado por la necesidad de una innovación permanente que parecía chocar con las poblaciones locales. ${ }^{7}$ Los técnicos enfrentan así la paradoja de estar atravesados por un discurso de cambio refractario a las prácticas locales, y por una demanda que les exige incorporarlas. En El Bolsón, otros especialistas reunidos para revisar la producción de fruta fina ${ }^{8}$ reconocieron que no existe un consenso técnico sobre la existencia o el potencial productivo de la frambuesa, producción tomada como central en la región desde la década de los sesenta.

Los cursos y encuentros resaltaron que el cambio es un horizonte cercano, la necesidad de mejora en la puesta en valor, o de un acceso a la comercialización no es sólo un objetivo del Estado, sino también de buena parte de los productores y pobladores. Bajo este contexto, la relevancia que localmente se da a la producción invisible (la del autoconsumo, el trueque, etcétera) choca con el ocultamiento que resulta de reducir la visibilidad a la capacidad de monetarizar lo que se considere productivo.

Así pues se puede pensar que la fuerza social se descubre estrictamente en momentos de emergencia, cuando es importante "eso" que acerca el vecino, amigo o familiar, y cuando el paisaje, antes que el desacuerdo referido, permite explicar la solidaridad. Pero esto tan relevante se pierde cuando la territorialidad de la región se inscribe en un programa de gobierno, donde "eso" común no forma parte de la política o de la planificación. Pero hay un elemento más. En el habitar la zona andina de Río Negro se desconoce la relevancia de la pertenencia provincial de forma sistemática (Núńez, 2016). Es como si la principal estructura administrativa que contiene a las localidades no tuviera

Las referencias tomadas de los cursos son menciones no literales. No acordamos compartir la literalidad de las menciones con cada autor/a, por ello sólo compartimos las referencias parciales que, como docentes, fuimos tomando en la serie de encuentros citados en el punto 3. Para preservar el anonimato, en caso de necesitarlo, referimos a cada persona con una letra, R. puntualmente es un/a extensionista de INTA, trabajando con rumiantes menores en la zona de estudio.

7 Cita tomada en curso de posgrado "Epistemología y Metodología de la Investigación Científica".

8 Tomado de la instancia de creación del "Observatorio de Políticas Públicas". 
peso, un aspecto que de hecho diferencia a Río Negro de otras provincias y que profundiza el marco de invisibilidad citado.

\section{Progreso, DeSARRollo E INNOVACiÓN EN LA REGIÓN ANDINA PATAGÓNICA}

Las nociones de "progreso", "desarrollo" e "innovación" se cruzan, sin embargo, son diferentes. Todas han sido referencia en los diseños e implementación de políticas económicas a lo largo del tiempo y de hecho, son términos que permiten un recorrido histórico. Temporalmente, la idea de "progreso" se presenta en los escritos sobre la región desde finales del siglo XIx hasta mediados del siglo xx. A partir de allí la noción de desarrollo comienza a regir como referencia hasta la década de los noventa, cuando la innovación emerge. La forma en que estos conceptos construyen sentidos territoriales y de ruralidad vincula las particularidades de la región a dinámicas globales base de las políticas de desarrollo.

\section{Progreso}

La norpatagonia argentina, desde su incorporación al Estado Nacional a partir del avance militar de finales del siglo XIx, fue descripta atendiendo al carácter autoevidente del progreso. Esta noción implicaba la presunción de un avance a una mejora estructural como destino ineludible (Navarro, 2004 y 2007). Núñez y Lema (2018) plantean que el progreso presumía que la condición del mismo, en la Patagonia, estaba dada por la destrucción de lo existente. Destacan una paradoja: las condiciones ambientales eran evidencia de un progreso ineludible, al tiempo que debían removerse personas, plantas y animales para su logro. Navarro (2011) señala que el desierto fue la referencia con que se caracterizó a la Patagonia desde las primeras menciones dentro de las esferas gubernamentales argentinas. Pero que antes que una caracterización ambiental, fue un programa de gobierno el que marcó la incorporación del territorio. Así, la falta de cultura fue el argumento del derecho de destrucción.

En este escenario, los sujetos del progreso-cambio no eran locales, sino que se remitían a capitales exteriores, que se suponían mejores para planificar por su vinculación al comercio internacional. En el proceso de conquista, la reflexión que inauguró el estudio científico que lo acompañó, señala que la exterminación de la población era condición necesaria para atraer a "la mas asustadiza de las asustadizas cosas del mundo, el capital destinado á vivificar 
las empresas de ganadería y agricultura..." (Ebelot, 1881, p. XI). De tal forma que el capital de inversión resultaba ser la naturaleza que debía preservarse y consolidarse en la región antes que cualquier otro elemento.

Coronato (2010) caracteriza al "modelo de ovinización" configurado a fines del siglo XIx para la Patagonia, y describe la introducción de enormes majadas de ovejas merino en las estepas, para propiciar la exportación de lana, dando lugar a una concentración de la propiedad de la tierra en grandes estancias.

Esta estructura económica inicial de la Patagonia contiene un carácter geopolítico fundamental, pues esta dinámica económica se constituyó vinculada al comercio trasatlántico con Gran Bretaña. Pero también en clave binacional, en tanto se asoció al modelo organizativo territorial del sur de Chile, también ligado a capitales foráneos, aunque mayormente germanos.

El crecimiento económico de la Patagonia andina argentina fue posible a partir de la articulación social y comercial con el dinamismo que estaba tomando el sur de Chile. Esta articulación ha sido explorada desde alianzas económico-familiares que unificaron en una misma zona económica a las áreas chilenas y argentinas colindantes propias de la cordillera (Méndez y Muñoz, 2013). Pero algo más, la vinculación se apoyó en una integración ambientalmente diversa, pues vinculó este dinamismo cordillerano con las producciones características de las áreas de estepa. Así se dio una articulación entre producciones ovinas clásicamente esteparias (Coronato, 2010), con madereras y agroganaderas, propias de la cordillera en estudio (Méndes, 2010; Méndez y Muñoz, 2013).

Todo este proceso tiene, a pesar de su magnitud, un carácter marginal. En los debates existentes sobre el modelo latifundista en el proceso por el cual la Patagonia se está integrando (Hora, 2019), la región no aparece a pesar de contener casi $60 \%$ del territorio argentino. Lo rural, en Argentina, se modeliza desde la estructura agroexportadora pampeana, en torno a la cual sí se debate, pero con una fuerza tal que eclipsa la particularidad del resto (Núñez y Michel, 2019). Así que, no sólo se trata de dar cuenta de una producción regional, sino de observar los ocultamientos que hablan de un modelo de nación en donde se inscribe lo rural.

La región en general se integra al país como área agrícola, sin mayores planificaciones, pero propiciando la introducción de cultivos y ganado a partir de la apertura en 1902 de las fronteras comerciales con Chile y facilitando que la producción se comercialice hacia el oeste (Méndez y Muñoz, 2013). La primera planificación fue llevada a cabo por el geólogo estadounidense Bailey Willis (1914), en la que proponía construir un Parque Nacional y una ciudad industrial en la zona de Bariloche, y lograr influencia en toda la zona andina. 
Este plan se abandonó ante la negativa del gobierno nacional por continuar con inversiones regionales, en parte por el resquemor de la relevancia que podría llegar a conseguir este espacio (Navarro, 2007). Sin embargo, la fuerza autoevidente del progreso como parte del paisaje queda explícita en la carta de despedida de Bailey Willis (1917) a su colaborador en el sur, Emilio Frey, cuando se le avisa del cierre de su proyecto: "Parece por el momento que nos detienen y nos obligan a parar en el trabajo para fomentar el progreso de las poblaciones sobre el lago..." comienza su carta. "Poco importa un presidente mientras que existan los recursos y las bellezas de la naturaleza, más atrayentes que jamás antes para las poblaciones cansadas de la guerra. 'Continuaremos sin desanimarnos', es lo que contesté al Sr. Dr. Ruiz Moreno y es el sentimiento sincero mío".

Continuar, desde su punto de vista, era dejar que la fuerza del paisaje demostrara la razón, que el progreso patagónico se impusiera desde el ambiente. Puede pensarse que algo de este orden impacta en la política pública al ver la constitución de áreas naturales en el país. En 1903 se reconoce la primera donación para la edificación de un Parque Nacional, al estilo americano, en la región. En el plan de Willis de 1914 se consideraba central la preservación de los bosques para el correcto desempeńo de la cuenca, y que propiciara el establecimiento de la infraestructura de riego, base de la explotación hidroeléctrica propuesta por él. Un aspecto interesante es que este cuidado no implicaba preservar especies, sino que sugería cambiar las plantas nativas por exóticas de crecimiento más rápido que dieran lugar a bosques más jóvenes (Willis, 1914, pp. 11-12). Willis repite la paradoja del progreso autoevidente, en una materialidad necesitada de remover lo nativo, aun apelando a la idea de crear un Parque Nacional.

En 1922 se crea el primer Parque Nacional, denominado Parque Nacional del Sud, ubicado alrededor del lago Nahuel Huapi, en lo que hasta entonces era la Colonia Agrícola Ganadera del Nahuel Huapi, hoy Bariloche. Esta iniciativa ligó el cierre de la frontera comercial con Chile a la conservación de bosques. Esta política se profundizó desde 1934, con la sanción de la Ley de Parques Nacionales, y la efectiva creación de los Parques Nacionales Nahuel Huapi e Iguazú, este último en el norte del país.

9 Bailey Willis llegó a Argentina en 1910 enviado por el Museo Nacional de los Estados Unidos para revisar los datos sobre la antigüedad del hombre en el Plata. Una vez instalado, fue contratado como Jefe de la Comisión de Estudios Hidrológicos de la Dirección General de Ferrocarriles, creada en 1911 para planificar el desarrollo de la norpatagonia. Esta comisión se cierra en 1915. Su carta de despedida, fechada en 1917, se encuentra en el Archivo Frey. Museo de la Patagonia de Bariloche. "Colección Frey". 
La región andina rionegrina, en los documentos estatales, parece reducirse a su paisaje en una retórica donde la importancia de la Patagonia radicaba en un valor estratégico que no necesariamente consideraba el crecimiento regional. Según Exequiel Bustillo, primer director de Parques Nacionales: "para mantener despierto y alerta el espíritu argentino, para eso y nada más que para eso, Dios ha colocado entre los peligros de la frontera las grandes bellezas de nuestra tierra" (Bustillo, 1946, p. 26). Una región que resultara educativa para la sociedad argentina en estos términos, atentaba contra el intercambio comercial local, basado en el modelo agrícola trasandino original (Méndez y Muñoz, 2013). El progreso, como urbanización o industrialización, se resolvió en escenarios alejados. Los Parques Nacionales y sus regiones aledañas se plantearon como márgenes que no tenían sentido sin ese centro y la actividad agrícola se planteó contradictoria a ese progreso nacionalista.

Cabe mencionar que el establecimiento de fronteras no fue una política argentina global, pues al norte de la cordillera patagónica no sólo no se establecieron superficies significativas de Parques Nacionales, sino que en la década de los treinta se promovieron los mayores intercambios comerciales por los pasos cordilleranos ubicados en la provincia de Mendoza (Lacoste, 2005), en la región cuyana del centro-oeste del país. Las montañas patagónicas quedaron como espacio de esparcimiento de sociedades urbanas que crecían en las metrópolis (Diegues, 2000; Fortunato, 2005), autoreferenciadas en un paisaje entendido como sublime y vacío.

Se tiene en esto otra paradoja geográfica, pues las montañas de la Patagonia son mucho más bajas que las de la zona cuyana, y los valles transversales tienen alturas inferiores que los hacen comparables a muchas rutas nacionales. Sin embargo, son estas montańas bajas las que se presentan como murallas infranqueables, mientras que los altos pasos del centro y norte de la cordillera de los Andes se ven como naturales para el comercio.

La década del treinta cambia la noción de progreso en la región andina y de estepa, la referencia se desliza desde las ovejas y los bosques hasta esa urbanidad lejana y tuteladora que se instala definitivamente como "lo natural". El progreso se definió desde el exterior, y el bien de Argentina se asoció a un cambio industrial y económico que se resolvía en escenarios no patagónicos. La sobre valoración del orden urbano estuvo apoyado en el imaginario de la naturaleza pura en las fronteras, como modo de justificar dinámicas que parecían antagónicas, pero que desde esta perspectiva resultaron funcionales al ordenamiento nacional en general (Serje, 2005). En Patagonia, el progreso apeló a la idea de falla autóctona. 


\section{Desarrollo}

El desarrollo, como objetivo de las políticas públicas, se instala a través del Estado planificador establecido por las presidencias de Juan Domingo Perón en el escenario argentino desde la década de los cuarenta (Berrotarán, 2003), y llega a la Patagonia en un contexto particular. En las décadas de los cuarenta y cincuenta la ganadería ovina fue perdiendo preeminencia, estableciéndose la fruticultura en el alto valle (véase figura 1) como base de la matriz económica norpatagónica (Bandieri y Blanco, 1998), en conjunto con la explotación petrolera que ya comenzaba a planificarse, aunque se afianzó en décadas posteriores (Favaro, 2001). Bajo el contexto del peronismo, con el objetivo de la industrialización, se demandaba la generación de energía. En este escenario, el plan de aprovechamiento hidroeléctrico (planteado como posibilidad desde el citado trabajo de Bailey Willis) se presentó en articulación con la estructura frutícola en desarrollo (Azcoitia y Núñez, 2013).

El caso de la construcción andina fue diferente, pues consolidó como centro al Parque Nacional Nahuel Huapi, reiterando el turismo como actividad central. Sin embargo, se sumó una paradoja. En el lago Nahuel Huapi, en la isla Huemul, se realizó un experimento nuclear, mismo que resultó un fracaso, pero que dio cuenta de la superposición de dos sentidos antagónicos generados desde el propio Estado: la repetición de un paisaje impoluto con la incorporación de una iniciativa con altos riesgos de contaminación (Mariscotti, 2005), que resultó ser la mayor inversión en la zona durante el periodo (Bessera, 2008).

En El Bolsón, durante las décadas cuarenta y cincuenta, se asentaron las condiciones que dieron lugar a una sistemática explotación maderera, frutícola y lupulera, que se desarrollará con claridad en la década de los sesenta (Méndes, 2010). En este proceso, la histórica vinculación de El Bolsón con la región de estepa se fue debilitando, planteándose un reconocimiento general llevado adelante desde la perspectiva con la cual se recorría la región de Bariloche.

De esta forma, los años cincuenta abren un nuevo proceso en la Patagonia por dos aspectos: por una parte, se reconocen los derechos políticos a partir de 1955, cuando se firma la provincialización de los territorios continentales del sur, ${ }^{10} \mathrm{y}$ por otra, el plan de diseño hidroeléctrico y explotación petrolera se dio

10 Los Territorios Nacionales fueron espacios incorporados tardíamente al Estado y tutelados desde el gobierno central. Esta figura se aplicó en las actuales provincias de Misiones, Chaco, Formosa, La Pampa, Neuquén, Río Negro, Chubut, Santa Cruz, Tierra del Fuego y un espacio denominado "Territorio de los Andes" entre las provincias de Salta y Jujuy. En 1955 se provincializan Neuquén, 
en el marco de las políticas reconocidas como desarrollistas (Rougier, 2016), desde las cuales se postularon intervenciones estatales con el objetivo de lograr el crecimiento económico regional, incorporando el término "desarrollo" como referencia ineludible de la política económica.

El desarrollo llega a la Patagonia con otra particularidad. La provincia, como ámbito administrativo, aparecía como respuesta a una larga demanda por derechos políticos. Río Negro, específicamente, se presenta desde los términos de su primer gobernador Edgardo Castello, al inicio investida de equidad. El primer gobernador asegura que su planificación provincial propiciaba un "desarrollo armonioso" que respondía a inequidades históricas que tenían a la línea sur como la principal víctima (García, 1960). Como estrategia se llevó adelante una inversión desigual que se pretendía reponiendo equidad, pero ello en lugar de generar equilibrios, profundizó diferencias regionales existentes. El resultado de su implementación en el tiempo fue el abandono de zonas rurales en las áreas de mayor vulnerabilidad (López, 2016; Herrera y Herrera, 2014), y la omisión de políticas específicas para la zona andina (Núñez, 2016).

Puntualmente, respecto al área de estudio, se observa una inversión económica prácticamente inexistente. Las producciones en la zona de El Bolsón se mencionan como potenciales, pero con una preponderancia menor respecto de otras regiones. Básicamente, durante décadas no se establecieron fondos para el desarrollo de cultivos o para mejorar la conectividad comercial, pues el paisaje de la zona de Bariloche se consideró tan imponente que se postuló que el área no necesitaba fondos, ya que resultaba atractiva para una inversión privada (Fantini, 1961). El paisaje es argumento, en la planificación provincial, para justificar la no inversión estatal en la vasta área de estudio.

El modo en que se instalaron las dos actividades principales de la región -turismo en Bariloche y agropecuario en El Bolsón- exponen una construcción provincial desigual, donde la idea misma del desarrollo se apoyó en desconocer las particularidades locales. A modo de ejemplo, la paradoja del turismo. Esta actividad llegó a Bariloche de la mano de una muy importante estructura nacional, la de los Parques Nacionales en la década de los treinta, que impactó en el ordenamiento de toda la región. Pero aunque desde la década de los cincuenta se implantó el turismo desde una escala nacional, y desde la escala provincial durante la década de los sesenta, no fue considerada

Río Negro, Chubut y Santa Cruz. También se provincializa Formosa. Chaco, La Pampa y Misiones habían sido provincializados previamente. El territorio de los Andes se había dividido entre Salta y Jujuy. Tierra del Fuego fue Territorio Nacional hasta 1992. 
actividad económica y, por tanto, adoleció de marcos de financiamiento y organización equiparables a otras actividades que generaban ingresos similares (Núñez, 2018).

Así, el centro económico de la región descansó sobre una actividad que, en términos de integración provincial, no fue considerada como productiva. Junto al eclipsamiento económico del turismo, la región perdió relevancia. Lo que ayuda a entender porqué entre los objetivos del gobierno provincial no estaba la articulación con la zona andina. Su actividad agrícola, también vista como casi inexistente, no contiene la misma valoración que las de otros espacios. Lo rural se promocionó en la zona irrigada del Alto Valle y Valle Medio, donde ya existía y que se tornó en el corazón de los procesos de exportación.

Se puede pensar que desde estos reconocimientos desiguales, la producción agropecuaria, vinculada a la localidad de El Bolsón, es doblemente oculta. Por un lado, por su negación en el marco del modelo de turismo, y por el otro, porque las actividades productivas tienen, en la provincia de Río Negro, un reconocimiento regional, y las actividades frutícolas fueron reconocidas como relevantes sólo en el Alto Valle y Valle Medio, y las hortícolas en el Valle Inferior.

En este periodo, además, es cuando en el marco del desarrollo nacional se crea el Instituto Nacional de Tecnología Agropecuaria (INTA), agencia del desarrollo agropecuario de escala nacional instalado en diferentes puntos del país. Uno de ellos se ubica en Bariloche en 1965, pero con una limitada articulación con la región aledaña. López (2016) señala que la localización en Bariloche tuvo como objetivo exclusivo la mejora ovina, que era una producción geográficamente alejada de este centro. La explicación de esta ubicación, para el autor, tiene que ver con la conformación de una red tecnocientífica nacional que, desde la década de los cincuenta toma a Bariloche como uno de sus puntos estratégicos. Así el INTA local se adhiere a una red tecnocientífica al tiempo que ignora la producción local. Ello no es menor si se recuerda que en la localidad de El Bolsón, durante esos años, se inició la producción comercial de fruta fina y se dio inicio a la explotación maderera a gran escala, así como a la producción de lúpulo financiada por la firma Quilmes (Méndes, 2010).

Todos estos procesos, de diverso origen y escala económica, impactaron en la dinámica de organización local, aunque su reconocimiento desde la escala provincial fue escaso y no tuvieron atención del INTA Bariloche hasta pasada la década de los setenta. Desde lo provincial, en la zona andina, el turismo se consideraba resuelto sólo por el paisaje (Núñez, 2018); el lúpulo generó un interés menor y la explotación maderera, así como la fruta fina casi no reciben atención en términos de política pública. La intervención estatal de 
nivel provincial, reflejada en las políticas de la época, repite la integración desigual como destino justificado en el paisaje. Localmente no hay productores relevantes. Los actores sociales, ajenos a las iniciativas del progreso, quedaron subalternizados en las políticas del desarrollo.

\section{Innovación}

En esta trayectoria, en los últimos 20 años, se comenzó a plantear la noción de innovación, asociada a cambios tecnológicos y de planificación con base en conceptos como ciudades inteligentes (Mitchell, 2007), plataforma territorial (Corrales et al., 2005; PROCIsUR, 2013), e incluso nuevas modalidades estructurales del capitalismos que están modificando las condiciones de periferia (Sztulwark, 2019). Lo cual lleva a preguntar por sentidos que cambian y permanecen respecto de los previos, es decir, los ecos de las nociones de progreso y desarrollo citadas.

Lattuada et al. (2015, p. 94), como síntesis de los objetivos de los programas de desarrollo rural en la última década, mencionan algunos que dialogan con el desarrollo en la región: i) la conformación de asociaciones formales de microproductores, que posibilitaron el fortalecimiento de unidades productivas, y mejorando la competitividad; ii) la innovación tecnológica en la producción, en la gestión y en la administración; iii) el mejoramiento de los canales de comercialización y de acceso a los mercados; iv) el acceso a información competitiva para la toma de decisiones, articulando su participación con clusters y organizaciones sectoriales.

En Patagonia estos objetivos se proyectaron en las poblaciones consideradas más vulnerables, con especial énfasis en jóvenes y mujeres, y de hecho se evaluaron como exitosos (Lattuada et al., 2015). Sin embargo, en la zona de estudio, la estructura de desigualdad regional y sectorial prácticamente no se ha modificado. Más bien se registra un desconocimiento sistemático hacia la producción vinculada a la agricultura familiar y a la producción doméstica, un aspecto reiterado en los estudios de la zona (Danklmaier et al., 2013; Cobelo, 2017; Valtriani, 2008), o a la producción de pequeña y micro escala en todos los sectores (Colino et al., 2016), que es la que contiene al principal número de habitantes.

Es interesante porque hay algo del orden del progreso que permanece. La principal trama de la producción es invisible, pues responde a la escala doméstica, que en muchos casos ni siquiera es reconocida en la categoría de productor, en tanto no destina su producción al mercado sino a la compleja 
trama de intercambios y solidaridades que se inscriben en la opaca categoría de "autoconsumo", con todas las problemáticas de reconocimiento ya vistas.

La primacía de la exportación, que aún se mantiene en los criterios de reconocimiento y en los indicadores de medición productiva, parece ser heredera de la primigenia visión de progreso, con la carga de destino que ello acarrea. El problema es que la innovación sigue tomando como ideal a los sectores dedicados al comercio exportador o de gran escala. Sin embargo, la trama desconocida de las producciones domésticas muchas veces ajenas a las dinámicas mercantiles, emerge como central en casos de emergencias, tanto socioambientales como económicas.

Así, desde la noción de innovación se podría pensar que no se han modificado los agentes del cambio. Los estereotipos reconocidos en el progreso se han replicado en la propuesta de innovación, sin ser nombrados. Esto impacta en la integración. Lo interesante de la actualidad, es que lejos de reconocer procesos globales, la innovación se apoya en estratégicas que se presentan como locales, casi como opciones individuales frente al camino aún abierto del desarrollo y el progreso, sin siquiera dialogar con las nuevas estructuras económicas mundiales. Los dinamismos globales, presentes en los periodos mencionados, se desdibujan, no por ser inexistentes sino por la ilusión del desacuerdo local como resultado de meras personas y no de historias políticas y económicas, que han estructurado las tensiones de la región y que operan como permanentes aún en el escenario de la innovación.

Con todo esto, la falta de datos sistemáticos sobre producciones familiares, regresan a la pregunta por las paradojas. La respuesta parecería estar en el reconocimiento de la agricultura familiar en todos sus diálogos escalares, una escala sobre la cual se ha escrito mucho pero que, aún en estudios actuales, se reconoce como ignorado en un área como la andina rionegrina, donde casi la totalidad de productores pertenece a dicha categoría.

\section{CONCLUSIONES}

Se puede sostener que las miradas sobre la producción e intervención actuales, ligadas a conceptos como innovación o diseño de territorios inteligentes, permiten reiterar la ilusión de un desarrollo rural sin sujeto, en tanto el reconocimiento de lo innovador se basa en lógicas, o bien empresariales, o bien científicas (Albornoz, 2009), opuestas a las valoraciones que se suponen estructurantes de la agricultura familiar. 
Las paradojas se definen a partir de que la medida de la pertenencia es el mercado. La inversión, la comercialización y la ganancia siguen siendo la medida central y exclusiva desde la cual se continúan estableciendo las mediciones de lo que existe.

A la luz de los resultados y solapamientos de distintas lógicas históricas, rastreables en la actualidad en la región andina de la provincia de Río Negro, se aprecia una articulación de la construcción de una región patagónica más amplia, así como con políticas globales. En más de 100 años se encuentra que el paisaje, como referencia del destino natural, se repite, naturalizando como actor del cambio a un tipo de productor particular, ligado a un comercio que, idealmente, excede el intercambio interno. A partir de las nociones de progreso y de desarrollo se evidencia una operación persistente, que refiere a la asignación de una carga peyorativa a las condiciones locales, como estrategia para justificar tanto los éxitos como los fracasos de las políticas delineadas.

El rastreo realizado aquí da cuenta de las huellas del progreso que persisten desde algunas lógicas provinciales de planificación. Aquellas ideas, que fueron parte del progreso, comandadas desde la escala nacional, hoy aparecen amalgamadas a la lógica provincial como en la ausencia de planificación provincial del desarrollo andino. La herencia de Río Negro como provincia que "desatiende la región andina", ${ }^{11}$ implica acuerdos con actores locales, pero sobre todo con una aceptación de esa desintegración como resultado de la geografía antes que de la política.

En este espacio andino la fuerte impronta nacional de Parques Nacionales operó como justificante de distancia. Desde aquí se actualiza la idea prístina de la naturaleza, que remite al orden nacional establecido a principios de siglo como si fuese resultado del propio paisaje. En los territorios de integración tardía, y sobre todo en aquellos investidos del imaginario de "naturaleza intocada" (Diegues, 2000), el deslizamiento de la política al paisaje permite justificar la integración desigual.

La noción de innovación atraviesa los programas de desarrollo de los últimos 20 años y Río Negro es una de las provincias que más intentó adherirse a la implementación de dichos programas e ideas. Sin embargo, la estructura de desigualdad no se ha modificado en tanto las herramientas de toma de datos siguen omitiendo referencias centrales a los actores de las escalas domésticas.

La innovación sigue reclamando como sujeto del cambio al actor económico delineado en el imaginario del progreso. En esta lógica la tensión entre lo

11 Mención repetida en entrevistas que abordan múltiples problemáticas (Núñez, 2016; Colino et al., 2016; Herrera y Herrera, 2014; López, 2016). 
local o autóctono sigue emergiendo, ya no en una clave explícitamente racista, pero sí siendo ignorado deliberadamente. Lo local queda fuera de los elementos del cambio, en una lógica que puede pensarse replicada en la consideración de la escala de la producción familiar.

Si se considerara una administración más amplia, la escala nacional, las herramientas de visualización del Estado no permiten salir de la valoración exclusiva a lo monetarizado. La herramienta censal podría ser un apoyo en tanto deje de ser una traba, los indicadores de producción y eficiencia de los distintos programas de promoción regional sumarían fuerza a la promoción local. El conocimiento de dinamismos propios sumaría a este camino. Lo doméstico, el autoconsumo, el intercambio, el mercado local, e incluso la visualización de la enorme movilidad regional, reconocidas en forma parcial, aparecen como una posible base de sistematización para pensar la innovación de forma real innovadora.

\section{BIBLIOGRAFÍA}

Albornoz, M. (2009), "Indicadores de innovación: las dificultades de un concepto en evolución", Revista Iberoamericana de Ciencia, Tecnología y Sociedad, vol. 5, núm. 13, Buenos Aires, Argentina, Centro de Estudios sobre Ciencia, Desarrollo y Educación Superior, noviembre.

Azcoitia, A. y Núñez, P. (2013), "Elementos de tensión en la planificación del desarrollo hidroeléctrico en Norpatagonia: 1911-1961", Revista de Historia Americana y Argentina, vol. 48, núm. 1, Mendoza, Argentina, Universidad Nacional de Cuyo, enero-junio.

Bandieri, S. y Blanco, G. (1998), "Pequeña producción, cambio productivo y capital británico en el alto valle de Río Negro (1900-1948)", Quinto Sol vol. 2, La Pampa, Argentina, Universidad Nacional de La Pampa, enerodiciembre.

Berrotarán, P. (2003), Del plan a la planificación. El Estado en la época peronis$t a$, Buenos Aires, Editorial Imago Mundi.

Bessera, E. (2008), Politicas de Estado en la norpatagonia andina. Parques Nacionales, desarrollo turístico y consolidación de la frontera. El caso de San Carlos de Bariloche (1934-1955), [Tesis de licenciatura en Historia], Universidad Nacional del Comahue, Argentina, Inédita, Repositorio Universidad Nacional del Comahue.

Bustillo, E. (1946), Los Parques Nacionales. Conferencia en el Salón Kraft, Buenos Aires, Editorial Kraft Ltada. 
Cobelo, C. (2017), Transformaciones territoriales en los Andes Patagónicos. El caso de las zonas rurales de El Bolsón, Río Negro, [Tesis doctoral en Ciencias Agropecuarias], Universidad de Buenos Aires, Argentina, Inédita, Repositorio Universidad de Buenos Aires.

Colino, E., Dondo, M. y Civitaresi, H. (2016), “Economía Social y Acción Colectiva. El caso de la economía barilochense de pequeña y micro escala”, Teuken Bidikay, vol. 8, Medellín, Colombia, Politécnico Colombiano, enero-junio.

Conti, S. y Núñez, P. (2012), "Poblaciones de la estepa rionegrina, el desafío de superar un pasado folklorizado y ser reconocidos como agentes económicos", Revista Artemis, vol. 14, núm. 1, Paraiba, Brasil, Universidad de Federal da Paraíba, enero-junio.

Coronato, F. (2010), El rol de la ganadería ovina en la construcción del territorio de la Patagonia, [Tesis doctoral], Paris тесH, Francia, <http://www.repositorio.cenpat-conicet.gob.ar/handle/123456789/970; jsessionid $=45 \mathrm{FE} 336$ 9968B317594670E43868DD81C>

Corrales, O., Carrera, F. y Campos, J. (2005), "El Bosque Modelo. Una plataforma territorial para la aplicación del enfoque ecosistémico", Recursos Naturales y Ambiente, núm. 45. Recuperado de <https://www.researchgate. net/publication/283266206_El_bosque_modelo_una_plataforma_territorial_para_la_aplicacion_del_enfoque_ecosistemico>

Danklmaier, C., Heinrichs, W. y Riveros, H. (2013), Activación territorial con Enfoque de Sistemas Agroalimentarios Localizados (AT-SIAL): La Comarca Andina del Paralelo $42^{\circ}$, Argentina-México, IICA.

Delgadillo, J. (2009), "Dimensiones territoriales del desarrollo rural en América Latina", Problemas del Desarrollo. Revista Latinoamericana de Economía, vol. 37, núm. 144, México, IIEc, <doi.org/10.22201/ iiec.20078951e.2006.144.7607>

Diegues, A. (2000), El mito moderno de la naturaleza intocada, Quito, Ecuador, Abya Yala.

Ebelot, A. (1881), "Introducción”, en A. Doering, Informe oficial de la comisión cientifica agregada al estado mayor general de la expedición al río Negro (Patagónia). Realizada bajo las órdenes del General D. Julio A. Roca. Entrega I-Zoología (pp. VII-XXIV), Buenos Aires, Osvaldo y Martínez.

Fantini, A. (1961), Provincia de Río Negro. Tres Años de Gobierno, Viedma, Secretaría de Relaciones Públicas.

Favaro, O. (2001), Estado, política y petróleo. La historia politica neuquina y el rol del petróleo en el modelo de provincia, 1958-1990, [Tesis Doctoral 
en Historia], Universidad Nacional de la Plata, Argentina, <http://www. memoria.fahce.unlp.edu.ar/library? $\mathrm{a}=\mathrm{d} \& \mathrm{c}=$ tesis $\& \mathrm{~d}=\mathrm{Jte} 131>$

Fortunato, N. (2005), "El territorio y sus representaciones como fuente de recursos turísticos. Valores fundacionales del concepto de "parque nacional", Estudios y perspectivas en Turismo, vol. 14, núm. 4, Buenos Aires, Argentina Centro de Investigaciones y Estudios Turísticos, octubre.

Garcés, P. (2019), "Of ends and means: Development policy assessment with human development and multiple causality", Revista Desarrollo y Sociedad 83, DOI <10.13043/DYS.83.10>

García, C. (1960), PRO 5. Programa de Desarrollo Económico de Río Negro 1960-1964, Viedma, Dirección General de Política Económica, Provincia de Río Negro.

Herrera, S. y Herrera, S. (2014), "Desequilibrios territoriales en la provincia de Río Negro: un análisis cuantitativo”, Pilquén, vol. 17, núm. 1, Viedma, Argentina, Universidad Nacional del Comahue, enero, <http://revele.uncoma.edu.ar/htdoc/revele/index.php/Sociales/article/view/1469>

Hora, R. (2019), "El debate sobre el latifundio en la Argentina de entreguerras", Historia Agraria, núm. 79, DOI <10.26882/histagrar.079e03h>

Klubock, T. (2014), La frontera. Forests and ecological conflict in Chile's frontier territory, Estados Unidos, Duke University Press.

Lacoste, P. (2003), "La crisis socioeconómica argentina y las respuestas sociales: las redes de clubes de Trueque", Confluencia, vol. 1, núm. 1, Mendoza, Argentina, Universidad Nacional de Cuyo, enero-junio, <http://m. bdigital.uncu.edu.ar/objetos_digitales/211/Lacoste.Confluencia1.pdf>

(2005), Argentina, Chile y sus vecinos, Mendoza, Andina Sur Caviar Bleu.

Lattuada, M., Nogueira, M. y Urcola, M. (2015), Tres décadas de desarrrollo rural en la Argentina: continuidades y rupturas de intervenciones públicas en contextos cambiantes 1984-2014, Buenos Aires, Teseo-UAI.

López, S. (2016), El INTA en Bariloche, una historia con enfoque regional, Viedma, Editorial UNRN.

Mariscotti, M. (2005), El secreto atómico de Huemul. Crónica del origen de la energía atómica en la Argentina, Buenos Aires, Estudio Sigma.

Méndes, J. (2010), Sociedades del bosque. Espacio social, complejidad ambiental y perspectiva histórica en la Patagonia andina durante los siglos XIX y XX, [Tesis Magíster en Ciencias Sociales], CLACso-flacso, Argentina, Inédita, Repositorio CLACSO-FLACSO.

Méndez, L. y Muñoz, J. (2013), "Alianzas sectoriales en clave regional. La norpatagonia argentino-chilena entre 1895 y 1920", en M. Nicoletti y 
P. Núñez (eds.), Araucanía-norpatagonia, la territorialidad en debate, San Carlos de Bariloche, IIDYPCA.

Michel, C., Núñez, P. y Easdale, M. (2020), "Censo y región en el reconocimiento agropecuario argentino", Cuadernos Geográficos, vol. 59, núm. 1, $<$ http://dx.doi.org/10.30827/cuadgeo.v59i1.8703>

Mitchell, W. (2007), “Ciudades inteligentes”, UOC Papers, núm. 5, Barcelona, España, Universitat Oberta de Catalunya, octubre.

Navarro, P. (2004), Patagonia, ciencia y conquista, Neuquén, Educo. (2007), Paisajes del Progreso, Neuquén, Educo.

(2011), "Territorios marginales: los desiertos inventados latinoamericanos. Representaciones controvertidas, fragmentadas y resignificadas", en D. Trejo (comp.), Los desiertos en la historia de América, México, usn H-UAC. Núñez, A., Aliste, E., Bello, Á. y Osorio, M. (2017), Imaginarios geográficos, prácticas y discursos de frontera: Aisén-Patagonia desde el discurso de la Nación, Chile, UCCH.

Núñez, P. (comp.) (2016), Sombras del desarrollo, Bariloche, IIDYPCA-UNRN. (2018), "Marcas y atractivos en una provincia imaginada. Río Negro, Argentina, 1958-1976”, Quinto Sol, vol. 22, núm. 1, DoI <dx.doi. org/10.19137/qs.v22i1.1208>

Núñez, P. y Lema, C. (2018), "Botánica, ciencia y guerra. Un análisis de los informes científicos de la 'Expedición al Río Negro (Patagonia)' de 1879”, Revista de Epistemología e Historia de la Ciencia, vol. 2, núm. 2, Córdoba, Universidad Nacional de Córdoba, junio-diciembre.

y Michel, C. (2019), "Territorios conquistados y trabajos invisibles. Orígenes del ordenamiento territorial patagónico", Pilquén, vol. 22, núm. 2, Viedma, Argentina, Universidad Nacional del Comahue, juniodiciembre, <http://revele.uncoma.edu.ar/htdoc/revele/index.php/Sociales /article/view/2273/pdf>

Oglietti, G. y Colino, E. (2011), "El ciclo de vida del destino turístico Bariloche. Particularidades del caso, limitaciones del enfoque y la contribución explicativa de la cepa turística de la enfermedad holandesa", III Congreso $A E D A$, Buenos Aires, 29, 30 y 31 de agosto, <http://www.memoria.fahce. unlp.edu.ar>

Otero, H. (1999), "Demografía política e ideología estadística en la estadística censal argentina 1869-1914”, Anuario IEHS 14.

PROCISUR (2013), El rol de los territorios en un marco de innovación y desarrollo, Buenos Aires-Montevideo, Procisur. 
prosap (2011), Plan de Desarrollo y Mejora Competitiva. Región Linea Sur de Río Negro, Buenos Aires, Ministerio de Agricultura, Ganadería y Pesca de la Nación.

Rougier, M. (2016), "Del peronismo al desarrollismo. La restricción externa y el debate sobre el capital extranjero", Revista de Ciencias Sociales, 91, Buenos Aires, Argentina, Universidad de Buenos Aires, enero-diciembre.

Serje, M. (2005), El revés de la nación, Bogotá, Uniandes.

Sztulwark, S. (2019), "La condición periférica en el nuevo capitalismo", Problemas del Desarrollo. Revista Latinoamericana de Economía, vol. 51, núm. 200, <doi.org/10.22201/iiec.20078951e.2020.200.68283>

Valtriani, A. (2008), Modelos de desarrollo forestal, sus conflictos y perspectivas en el sector de micro PyMES forestales; estudios de caso en la región noreste y centro de la provincia de Chubut, [Tesis doctoral en Economía], Universidad de Buenos Aires, Argentina, Inédita, Repositorio Universidad de Buenos Aires.

Willis, B. (1914), El norte de la Patagonia. Naturaleza y riquezas. Estudio de los elementos del tráfico del ferrocarril nacional de fomento desde Puerto San Antonio hasta el lago Nahuel Huapi y sus ramales dentro de la cordillera hasta su extensión internacional con término en Valdivia en Chile, New York, ScribnerPress. 